\title{
European urologists prefer surgery for high-risk prostate cancer
}

Results of a web-based survey provide insight into the practice patterns of European urologists managing men with high-risk prostate cancer; radical prostatectomy was the first-line treatment of choice for $60 \%$ of respondents.

In the absence of a consensus regarding standard of care for men with high-risk prostate cancer, members of the Prostate Cancer Working Group of the Young Academic Urologists Working Party of the EAU put together an online survey to collect urologists' preferences. They invited 12,850 physicians-including 8,234 European urologists-to complete their questionnaire of 29 items by email.

Data were analysed after 30 days; 585 urologists from across Europe completed the survey. The investigators report a high level of agreement (67\%) for the definition of high-risk prostate cancer (PSA $\geq 20 \mathrm{ng} / \mathrm{ml}$, clinical stage $\geq \mathrm{T} 3$ or biopsy Gleason score $\geq 8$ ). Initial radical prostatectomy (as part of a multimodal treatment regimen) was the preferred treatment option of $60 \%$ of respondents; $29 \%$ preferred external beam radiation therapy with androgen deprivation therapy and $4 \%$ chose radical prostatectomy as monotherapy.

Regarding surgical approach, open retropubic surgery was the most popular option (60\%), followed by robot-assisted surgery (20\%) and laparoscopic surgery (15\%). 96\% of respondents routinely perform pelvic lymph node dissection.

Despite the low response rate $(7 \%)$ of this survey, the results certainly provide some interesting points of discussion regarding the management of high-risk prostate cancer.

\section{Sarah Payton}

Original article Surcel, C. I. et al. Preferences in management of high-risk prostate cancer among urologists in Europe: results of a web-based survey. BJU Int. doi:10.1111/bju.12796 